Crouch, C. (2008). Postdemokratie. Frankfurt/M: Suhrkamp.

De Souza Briggs, J. (2008). Democracy as Problem Solving. Civic Capacity in Communities Across the Globe. Cambridge: MIT Press.

Dewey, J. (2001). Die Öffentlichkeit und ihre Probleme. Berlin: Philo. Lathrop, D.; \& Ruma, L. (2010). Open Government: Collaboration, Transparency, and Participation in Practise. Sebastopol, CA: O'Reilly.

Leggewie, C. (2011). Mut statt Wut. Aufbruch in eine neue Demokratie. Hamburg: Körber Stiftung.

Nanz, P.; \& Fritsche, M. (2012) Handbuch Bürgerbeteiligung. Verfahren und Akteure, Chancen und Grenzen. Bonn: Bundeszentrale für politische Bildung.

Olte, P. (2012). Was ist Demokratie? Geschichte und Gegenwart. München: C.H. Beck.

Sartori, G. (2006). Demokratietheorie. 3. Aufl. Wiesbaden: Wissenschaftliche Buchgesellschaft.

Schnelle, K.; \& Voigt, M. (2012). Energiewende und Bürgerbeteiligung: Öffentliche Akzeptanz von Infrastrukturprojekten am Beispiel der ,Thüringer Strombrücke“. verfügbar unter: http://germanwatch.org/en/download/4135.pdf.
Versteyl, A. (2001). Partizipation durch Verfahren: Verbesserung der Öffentlichkeitsbeteiligung auf Grundlage der gesetzlichen Regelungen.I $+E-$ Zeitschrift für Immissionsschutzrecht und Emissionshandel, 89-96.

Der Text ist eine gekürzte Version des Policy Briefs „Deutschlands Energiewende-Demokratie kollaborativ gestalten“, der von der Projektgruppe „Kollaborative Demokratie“ der stiftung neue verantwortung (www.stiftung-nv.de) erarbeitet wurde. Das 10-köpfige Team aus Wirtschaft, Wissenschaft, Politik und Beratung trieb die Frage nach der Zukunft der Demokratie um. Im Mittelpunkt der Arbeit stand die Frage: Wie bringen wir den wachsenden Wunsch nach direkter Beteiligung mit dem praktischen Bedürfnis nach politischer Handlungsfähigkeit in Einklang?

\title{
Zum dauernden Besitz verfasst: Thukydides“ „Geschichte des Peloponnesischen Krieges" als Klassiker der Politikberatung
}

Andreas Lotz

\begin{abstract}
Kernaussagen:
Ist das Werk des antiken Historikers Thukydides für die Politikberatung von Bedeutung? Dagegen spricht auf den ersten Blick mehr als nur die Tatsache, dass es sich hierbei um ein Geschichtswerk handelt. An der wechselvollen Rezeption seines Werkes wird ebenfalls deutlich, dass sich dessen politikberaterische Relevanz nicht unmittelbar erschließt. Und doch fördert eine konsequente Lektüre das politikberaterische Potential dieses umfangreichen Werkes zu Tage.
\end{abstract}

\section{Einleitung}

Aristoteles spricht in seiner „Poetik“ der Geschichtsschreibung die Fähigkeit ab, Aussagen über das Allgemeine treffen zu können. Die Aufgabe der Dichtung sieht er hingegen darin, „das nach den Regeln der Wahrscheinlichkeit oder Notwendigkeit Mögliche“ (1451 b) in Worte zu fassen. Anders als der Geschichtsschreiber, der bloß über das wirklich Geschehene berichte und nur das Besondere behandle, teile der Dichter mit, was geschehen könnte und sei daher in der Lage über das Allgemeine zu sprechen. Die Poesie, urteilt Aristoteles, sei somit im Unterschied zur Geschichtsschreibung etwas Ernsthafteres und Philosophischeres.

Inwiefern trifft diese Auffassung auf Thukydides' Darstellung des sogenannten Peloponnesischen Krieges ${ }^{1}$ nicht zu? Sein Gegenstand ist zwar ein spezieller - eine langjährige krie- gerische Auseinandersetzung -, der Anspruch jedoch, den er erhebt, geht darüber hinaus. Das bezeugt folgende Aussage am Ende des sogenannten Methodenkapitels:

„Zum Zuhören wird vielleicht diese undichterische Darstellung minder ergötzlich scheinen; wer aber das Gewesene klar erkennen will und damit auch das Künftige, das wieder einmal, nach der menschlichen Natur, gleich oder ähnlich sein wird, der mag sie so für nützlich halten, [...]: zum dauernden Besitz nicht als Prunkstück fürs einmalige Hören ist sie verfasst" (I, 22).

Obzwar Thukydides dezidiert erklärt, dass sein Werk nichts mit Dichtung gemein habe, erfüllt es dennoch Aristoteles' Kriterien. Es beansprucht nämlich für sich, allgemeingültige Erkenntnisse präsentieren zu können, die nicht nur das Vergangene betreffen, sondern auch in Zukunft relevant sein werden. Indem der Historiker die Nützlichkeit seiner Erkenntnisse anspricht, enthüllt er die didaktische Absicht seines Werkes. Durch die Formulierung, seine Schrift sei kein Prunkstück, sondern zum dauernden Besitz verfasst, bringt Thukydides

1 Thukydides“ Vereinheitlichung der aufeinander folgenden Kriege zwischen Athen und Sparta - der erste Krieg endete mit einem Friedensvertrag im Jahre $423 \mathrm{v}$. Chr. und wurde offiziell erst zehn Jahre später aufgenommen - zu einem einzigen Konflikt erschien seinen Zeitgenossen mit ziemlicher Sicherheit als fragwürdig. Deshalb erklärt er nach der Darstellung des Friedensschlusses: „Insgesamt dauerte damit der Krieg siebenundzwanzig Jahre. Denn die Einigung zwischenhinein nicht als Krieg bezeichnen zu wollen, wäre ein Irrtum: man achte auf die Tatsachen, wie eins das andere ablöste, und man wird finden, daß das nicht wohl Frieden heißen darf" $(V, 26)$. Zitiert wird im Folgenden nach Thukydides (1976). 
zum Ausdruck, dass die darin enthaltenen Erkenntnisse zum Gebrauch gedacht sind. Er deutet damit an, dass sein Werk für die politische Praxis relevant sein könnte.

Er selbst bekleidete nur für kurze Zeit ein Strategenamt und war aufgrund der Verbannung aus Athen gezwungen, das politische Geschehen über einen langen Zeitraum aus der Äquidistanz zu den am Krieg beteiligten Parteien zu beobachten. Er hatte mithin verhältnismäßig wenig politische Erfahrung und war, soweit bekannt, nie als politischer Berater tätig. Wenngleich diese Tatsachen die politikberaterische Relevanz seines Werkes auf den ersten Blick schmälern mögen, ist die Frage, ob seine Darstellung jenes gewaltsamen Konflikts als ein Klassiker der Politikberatung gelten könnte, damit nicht erledigt. Zunächst: Was spricht dagegen und wie verlief die Rezeption des Thukydides?

\section{Kein Klassiker der Politikberatung?}

Gegen die Erhebung des Thukydides zum Klassiker der Politikberatung kann erstens eingewendet werden, dass sein Werk keine genuin politikberaterische Abhandlung, sondern erklärtermaßen eine historische Darstellung von Kriegsereignissen ist, aus der sich, zweitens, nicht umstandslos politische Handlungsmaximen ableiten lassen. Denn anders als im „Il principe“ Niccolò Machiavellis, der Thukydides in wichtigen Aspekten nahe steht (vgl. Münkler, 1984, S. 261 f.), finden sich beim antiken Historiker weder ausformulierte Gebrauchsanweisungen noch feste Lehrsätze. Drittens, fällt Thukydides' Schweigen bezüglich der aus seiner Darstellung zu erschließenden Konklusionen auf, was immer wieder Kontroversen über das Sinnganze seines Werkes genährt hat (Meineke, 2003, S. 481 f.). Hinzu kommt, viertens, dass er, im Gegensatz zu Machiavelli, nicht dezidiert darlegte, für welches Publikum er schrieb. Fünftens trug sein oftmals schwer verständlicher Stil sicherlich dazu bei ${ }^{2}$, im Unterschied zur klaren Sprache Machiavellis, dass sein Werk selten zur praktischen Orientierung herangezogen wurde.

Dies sowie die Tatsache, dass Thukydides die Frage nach der ,besten Staatsform‘ unbeantwortet ließ, führte dazu, dass er in der Neuzeit - nach seiner Wiederentdeckung in der Renaissance - fast ausschließlich von Historikern und Philosophen gelesen wurde (vgl. zum Nachfolgenden ebd.). So wirkte sich der Umstand, dass er ein Krisenautor war, wenig auf die Rezeption seines Werkes aus. Denn es war vor allem Tacitus, der im Zeitalter der politischen Konflikte, die Europa seit Mitte des 16. Jahrhunderts erschütterten, zur politischen Belehrung heran gezogen wurde. Der religiös gefärbte Charakter der damaligen Kriege sowie das Aufkommen des Absolutismus machten es den Rezipienten zunehmend schwerer, Parallelen zu ihrer Gegenwart im Werk Thukydides' zu finden.

Diese sozio-politischen Erschütterungen schwächten jedoch das moralpädagogische historische Interesses allmählich $a b$, das „durch eine auf die Feststellung konstanter Daseinsprinzipien gerichtete philosophische Reflexion der Geschichte abgelöst wurde“ (ebd., S. 487). Thomas Hobbes und andere neuzeitliche Denker nahmen nunmehr die spezifi- sche Erkenntnisleistung des Thukydides würdigend wahr. Die Diskussion um die US-amerikanische Bundesverfassung wurde mit Bezug auf dessen pessimistische Anthropologie und die Darstellung des Niedergangs der Athener Demokratie als abschreckendem Musterfall geführt. Im 20. Jahrhundert, dem „Zeitalter der Extreme“ (E. Hobsbawm), wurde der antike Geschichtsschreiber in den verschiedensten Variationen von den unterschiedlichsten Parteien für die eigene politische Sache instrumentalisiert. Nach dem II. Weltkrieg erklärte die Denkschule des sogenannten Realismus innerhalb der Internationalen Beziehungen Thukydides zu einem ihrer wichtigsten Vordenker (dazu Johnson Bagby 1994).

\section{Zur politikberaterischen Relevanz Thukydides“}

Um Thukydides‘ politikberaterische Relevanz, die sich nicht unmittelbar aufdrängt, erschließen zu können, bedarf es einer sorgfältigen Lektüre. Denn er operiert nicht mit einprägsamen Formulierungen, sondern bedient sich der sophistischen Methode der Antilogien. Indem er antithetische Positionen in

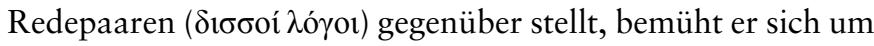
eine polyperspektivistische Darstellung bestimmter Konstellationen und Probleme. Er platziert die Reden dort, wo die Fäden des Geschehens zusammenlaufen und ein repräsentativparadigmatisches Moment vorliegt. Die Reden stehen an denjenigen Stellen, die zur Rück-, Um- und Vorschau animieren. Und sie enthalten bestimmte Gedankenkomplexe, die wiederholt von denselben oder verschiedenen Sprechern vorgetragen werden. Durch die Wiederaufnahme analoger Gedankengänge in disparaten Situationen wird eine Verknüpfung zwischen einmaligem Geschehen und allgemeinen Prinzipien hergestellt (vgl. Regenbogen, 1933).

\subsection{Týche}

Solch ein allgemeines Prinzip der historisch-politischen Abläufe ist $\tau$ $\chi \eta$, die in einer gegebenen Situation eigentlich nur negativ zu fassen ist, insofern Thukydides mit diesem Wort das bezeichnet, „was dem planenden und handelnden Menschen sich nicht oder nicht mehr fügen will“ (Müri, 1947, S. 254, Hervorhebung im Original). Damit benennt er all das,

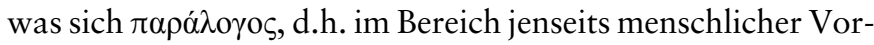
aussicht und Berechenbarkeit, befindet. Er gemahnt daran, dass es das Unverfügbare gibt - beispielsweise Naturereignisse (I, 23, 101; II, 8, 77; III, 49, 89; IV, 3; V, 45; VI, 70, 95; VII, 50, 79) -, das sich im Unterschied zum eigenen Entschluss nicht beherrschen lässt (IV, 64), und dass die Möglichkeiten des Menschen mithin nicht grenzenlos sind. Die Querschläge der týche können nicht berechnet (VI, 78), aber beim Entwerfen von Strategien mit eingerechnet werden (vgl. Herter, 1950, S. 140). Das Vermeiden von Risiken gehört demnach zum vernunftgeleiteten Planen dazu (VI, 23), darf aber nicht mit Inaktivität verwechselt werden oder zu Passivität führen (II, 87).

2 Bereits in der Antike bekunden die Rezipienten ihre Schwierigkeiten mit Thukydides' Sprache (vgl. Regenbogen, 1933, S. 3 f.). 
Das Einkalkulieren der týche in die eigenen Pläne ist zudem nicht gleichbedeutend mit dem bloßen Vertrauen auf ihre Macht. Denn sie begünstigt nie nur die eine Seite (VII, 61; vgl. auch IV, 18). Deshalb ist es verheerend, wenn aus blinder Zuversicht auf die týche heraus gehandelt wird (siehe dazu III, 97).

So betont der spartanische König Archidamos gegenüber seinen Landsleuten, dass man bei einer bevorstehenden Auseinandersetzung weder auf die Fehler des Feindes hoffen noch seine geistigen Fähigkeiten unterschätzen, sondern die eigene Strategie ausgehend von der Annahme entwickeln sollte, dass die gegnerischen Pläne durchdacht entworfen wurden (I, 84, vgl. zudem II, 11; VI, 11). Die gleiche Einsicht spricht Perikles in einer für Athen kritischen Situation aus, als er versucht, aufgebrachte Bürger zu beruhigen und ihnen Mut zuzusprechen:

„[D]er Wagemut bekommt mehr Festigkeit, wenn, bei gleicher Gunst des Glücks, die Vernunft sich überlegen füblt; sie vertraut auch nicht dem Hoffen (das in der letzten Not seine Kraft zeigt), sondern der mit dem Gegebenen rechnenden Erkenntnis, deren Voraussicht verläßlicher ist" (II, 62).

An den Ausführungen zur týche wird ersichtlich, dass Thukydides - ohne es ausdrücklich zu erklären - sich insbesondere an die okkasionell-aktiven politischen Akteure wendet (vgl. Regenbogen, 1933, S. 30). Er demonstriert, dass eine rationellpraktische Bewältigung anfallender Aufgaben und drängender Probleme stets gefährdet ist, sei es durch das jede Berechnung durchkreuzende Walten der týche, sei es durch die Zwangsläufigkeit politischer Entwicklungen.

\subsection{Anánke}

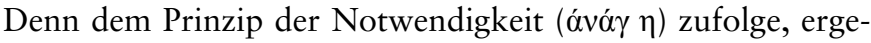
ben sich aus bestimmten Entscheidungen oder Reaktionen nicht nur Handlungsalternativen, sondern notwendigerweise auch Konstellationen von Verhältnissen, die Zwänge für die politischen Akteure mit sich bringen. So hat die Zurückhaltung der Spartaner im Kampf gegen das Perserreich nach Ansicht der Athener dazu geführt, dass sie in die Führungsrolle geradezu gedrängt wurden: die Übernahme der Führungsposition durch Athen stellte den ersten Schritt Richtung Vormacht dar. Die Athener betonen, dass sich aus dieser machtpolitischen Verschiebung notwendigerweise weitere Schritte ergaben. Anders ausgedrückt: sie konnten nicht umhin, ihre Dominanz weiter auszubauen, wollten sie ihre politische Existenz nicht gefährden (I, 75; vgl. Herter, 1950, S. 143). ${ }^{3}$ Die veränderte machtpolitische Lage wirkte sich ihrerseits auf die außenpolitische Wahrnehmung der Spartaner aus und musste zwangsläufig zu einer Gegenreaktion führen. ${ }^{4}$ Thukydides schreibt diesbezüglich, dass die öffentlich vorgebrachten Beschuldigungen seitens Spartas nur Anlässe für eine Kriegserklärung waren. Die eigentliche Ursache des Krieges sei die durch Athens Expansion hervorgerufene Furcht der Spartaner, die sie zu einer militärischen Reaktion gezwungen habe (I, 23, vgl. zudem I, 88, 118).

\subsection{Der Mensch als politisches Wesen}

Furcht ist Bestandteil der affektiven Verfasstheit des Menschen, der als Träger des geschichtlichen Geschehens bei Thukydides in dreifacher Gestalt dargestellt wird: als Individuum, als Typus und als Kollektiv (vgl. dazu Regenbogen, 1933, S. 10-17). Thukydides stattet die politischen Akteure nur in wenigen Fällen mit individuellen Zügen aus und lässt sie vor allem als Typen auftreten. Dabei stilisiert er sie zu Exponenten einer bestimmten politischen Haltung oder zu Musterexemplaren einer Politikergattung. Indem er unterschiedliche Typen von politischen Akteuren konturiert darstellt, vermag er zugleich anhand ihrer Reaktionen in bestimmten Lagen verschiedene Handlungsmöglichkeiten für ähnliche Situationen vorzuführen. Statt daraus fest umrissene Handlungsmaximen abzuleiten, regt er mittels ausführlicher Beschreibung der Handlungsfolgen zur eigenständigen Reflexion an (ähnlich ebd., S. 9 f., 17).

Politische Aktivitäten erschöpfen sich jedoch nicht in Auseinandersetzungen einzelner politischer Akteure, sondern betreffen notwendig das gemeinsame Handeln von Kollektiven. Thukydides' Analyse kollektiver Dynamiken setzt beim synchronen Vergleich zwischen Athen und Sparta an: die späteren Kriegsgegner werden aus der Außenperspektive der Korinther als höchst verschiedene politische Einheiten dargestellt. Dabei charakterisieren sie die Spartaner als zögerlichzurückhaltend, während die Athener als risikofreudig und innovativ beschrieben werden (I, 68-71; zudem VIII, 96). ${ }^{5}$ In ihrer anschließenden Verteidigungsrede versuchen die Athener aus der Innensicht heraus geschichtlich zu begründen, wie sie zu ihrer militärisch-wirtschaftlichen Überlegenheit gelangt sind (I, 73-75). Ihre diachrone Darstellung offenbart, dass Athens und Spartas unterschiedliche ökonomisch-politische Herangehensweisen und Mentalitäten, die eine strukturelle Wirkung auf das Handeln der jeweiligen Akteure haben, das Ergebnis eines historischen Prozesses sind. Thukydides gemahnt mithin an die strukturellen Zwänge, zeigt aber implizit, dass es alternative Entwicklungsmöglichkeiten gibt. Er verdeutlicht zudem, dass bestimmte Eigenschaften, die in gewissen Situationen bzw. Konstellationen zum Erfolg führen, nicht in jeder Lage vom Vorteil sind. So betont Perikles, dass der Drang, stets das Erreichte überschreiten zu wollen, den er als ein positives Merkmal athenischer Mentalität herausstellt (II, 39 f.), im Krieg gegen Sparta nicht das Handeln Athens bestimmen darf (I, 144).

Gleichwohl gibt es eine Konstante - Athener führen sie apologetisch an (I, 76; ähnlich V, 105) -, die beim Analysieren und Planen stets mit einzubeziehen ist: die menschliche Natur.

3 Alkibiades verweist auf dieselben ,Zwänge“ als er seine Mitbürger zur militärischen Aktion gegen Sizilien animiert (VI, 18). Ähnliche Argumentation findet sich außerdem in Kleons Rede während der athenischen Debatte über Mytilene (III, 40).

4 Perikles erkennt ebenfalls, dass der Krieg notwendig kommen wird und empfiehlt deshalb den Athenern, als Ausdruck des richtigen Umgangs mit der anánke, diese Tatsache aktiv anzunehmen (I, 144).

5 Vgl. ferner zum spartanischen Zaudern bzw. Mangel an Übermut I,118; II, 94 und zu Athens Drang-nach-mehr IV, 21, 41. 
Thukydides erklärt unmissverständlich, dass zum Wesen des Menschen die Ehrsucht, das Streben nach eigenem Nutzen ${ }^{6}$ sowie das Mehrhabenwollen gehört. Er exponiert ferner die Furcht als den markantesten Ausdruck emotionaler Instabilität und stellt kollektive Gefühlsschwankungen als eine Herausforderung an politische Führung dar (I, 140; II, 59, 61, 65; III, 36). ${ }^{7}$ Da die Affekte und Leidenschaften bemerkenswert häufig das Handeln der kollektiven Akteure bestimmen, ist das Movens der Politik nicht immer in der Verfolgung rationaler Interessen zu suchen. Unfehlbares Kalkül ist demnach eher selten möglich.

\section{Schlussbetrachtung}

Die notwendige Voraussetzung des erfolgreichen politischen Handelns ist daher cíkós, die Fähigkeit verschiedene Aspekte und Erfahrungen zur Erstellung von Prognosen oder Strategien miteinander verbinden und vergleichen zu können. Es ist ein Denken, das sich am Wahrscheinlichen orientiert (vgl. Hunter, 1973, S. 25-41). Wie funktioniert es?

Der Plan Archidamos“ ist augenscheinlich wohlüberlegt: Er schätzt die wirtschaftliche Stärke und die militärischen Kapazitäten Athens als beträchtlich ein (I, 80, 81, 83). Er erkennt implizit das, was Perikles explizit ausdrückt (I, $141 \mathrm{f}$.): dass im Falle einer langfristigen Auseinandersetzung Athen aufgrund ökonomischer Überlegenheit strategische Vorteile hat. Sein Vorgehen besteht daher darin, Athen rasch zu einer Schlacht zu bewegen. Er bezieht kollektive Emotionalität als Faktor in seine Strategie ein, indem er davon ausgeht, dass die Verwüstung des Athener Umlandes sich als ein affektiver Anstoß erweisen wird: emotional erschüttert, zumal sie solche Zerstörung vor den eigenen Toren nicht kennen, werden die Athener unüberlegt handeln und sich den Spartanern stellen (II, 11). Dass es dazu nicht kam, liegt nicht nur daran, dass die Spartaner zu lange Oinoë belagerten, sondern resultiert vor allem aus der Fähigkeit Perikles', mit der affektiven Aufgeladenheit seiner Mitbürger umzugehen, die in der Tat aufgebracht waren und ausrücken wollten (II, 18-22). Archidamos' Strategie bewährte sich demnach nicht, weil er den Faktor politische Führung ignorierte. Welche Lehren lassen sich daraus und aus Thukydides' Werk überhaupt ziehen?

Die Orientierung am Wahrscheinlichen setzt ein ausgewogenes Vertrauen in die eigenen Fähigkeiten voraus. Dazu gehört zudem, in einer gegebenen Situation die Verhältnisse adäquat erfassen und sich entsprechend verhalten zu können. „Wer seinesgleichen nicht nachgibt, dem Stärkeren wohl begegnet, gegen Schwächere Maß hält, der fährt meist am besten “ (V, 111). So lautet der Ratschlag der überlegenen Athener an die Melier. Dass sie sich selbst an ihn letztlich nicht hielten, spricht angesichts ihres Desasters auf Sizilien für seine Richtigkeit (vgl. VII, 42-87). ${ }^{8}$ Sich-am-Wahrscheinlichen-orientieren bedeutet ferner, alle erdenklichen Aspekte und Elemente einer Konstellation unbedingt zu berücksichtigen und zugleich mit den Querschlägen der týche zu rechnen, die Möglichkeit des eigenen Scheiterns in Betracht zu ziehen.

Das Einkalkulieren jedweder Faktoren und Perspektiven erscheint im „Zeitalter der Beschleunigung“ (H. Rosa) als ein ebenso unmögliches Unterfangen wie die Orientierung am voluminös-umständlichen Werk von Thukydides. Gleichwohl lohnt sich die Mühe einer geduldig-genauen Lektüre dieses Werkes, das fraglos das Potential zum Klassiker der Politikberatung hat. Aristoteles irrte sich: die Geschichtsschreibung vermag allgemeingültige und praktikable Erkenntnisse bereitzustellen. Thukydides liefert dafür einen eindrucksvollen Beleg. Nicht umsonst wurde sein Werk zum dauernden Besitz verfasst.

\section{Literatur}

Aristoteles (1982). Poetik. Stuttgart: Reclam.

Johnson Bagby, L. M. (1994). The Use and Abuse of Thucydides in International Relations. International Organisation, 48 (1), 131-153.

Herter, H. (1950). Freiheit und Gebundenheit des Staatsmannes bei Thukydides. Rheinisches Museum für Philologie, 93, 133-153.

Hunter, V.J. (1973). Thucydides: The Artful Reporter. Toronto: Hakkert.

Meineke, S. (2003). Thukydidismus. In Der Neue Pauly: Enzyklopädie der Antike (Bd. 15/3, S. 480-494). Stuttgart, Weimar: Metzler.

Münkler, H. (1984). Machiavelli: Die Begründung des politischen Denkens der Neuzeit aus der Krise der Republik Florenz. Frankfurt a. M.: Fischer.

Müri, W. (1947). Beitrag zum Verständnis des Thukydides. Museum Helveticum, 4, 251-275.

Regenbogen, O. (1933). Thukydides als politischer Denker. Das humanistische Gymnasium, 44, 2-25.

Thukydides (1976). Geschichte des Peloponnesischen Krieges (2., überarb. Aufl.). Zürich, München: Artemis.

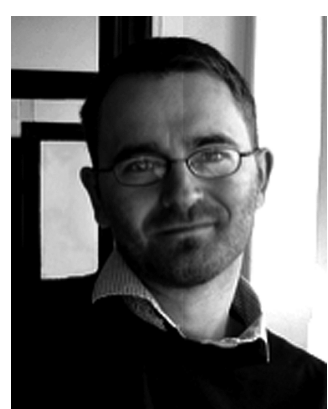

Studium der Geschichte und Politikwissenschaft in Wien, Riga und Berlin. Seit 2010 wissenschaftlicher Mitarbeiter am Sonderforschungsbereich 644 „Transformationen der Antike" der Humboldt-Universität zu Berlin. Forschungsschwerpunkte: Imperien, Politische Theorie und Ideengeschichte, Politische Philosophie, Psychoanalyse und Politik. Email: andreas.lotz@sowi.hu-berlin.de

6 So kommt moralisch-rechtlichen Überlegungen in den Reden eine geringere Bedeutung gegenüber den Appellen an den Eigennutz der Zuhörer zu. Vgl. dazu die Debatte über Mytilene, in der primär vom Nutzen für Athen her argumentiert wird (III, 37-48). Vgl. ferner I, 32; III, 14 ; VI, 83, 86, 87 .

7 Zur massenpsychologischen Wirkung des Aberglaubens vgl. VI, 70; VII, 50,79 .

8 Siehe zudem die falsche Einschätzung der Macht Athens durch die Chier (VIII, 5-8, 14 f., 22-24). 